\title{
Limited evidence suggests no difference in orthodontic attachment failure rates with the acid-etch technique and self-etch primers
}

\section{Abstracted from \\ Hu H, Li C, Li F, Chen J, Sun J, Zou S, Sandham A, Xu Q, Riley P, Ye Q.}

Enamel etching for bonding fixed orthodontic braces. Cochrane Database Syst Rev. 2013; 11: CD005516. DOI: 10.1002/14651858.CD005516. pub2

Address for correspondence: Cochrane Oral Health Group, School of Dentistry, The University of Manchester, Coupland 3 Building, Oxford Road, Manchester, UK. M13 9PL E-mail: cohg@manchester.ac.uk

\section{Question: What is the best dental etchant and etching technique for bonding-fixed orthodontic appliances?}

Data sources The Cochrane Oral Health Group's Trials Register, the Cochrane Central Register of Controlled Trials (CENTRAL), Medline, Embase, Chinese Biomedical Literature Database, the WHO International Clinical Trials Registry, and the National Institutes of Health Clinical Trials Registry were searched with no restrictions regarding language or date of publication.

Study selection Randomised controlled trials comparing different etching materials, or different etching techniques using the same etchants, for the bonding of fixed orthodontic brackets to incisors, canines and premolars in children and adults.

Data extraction and synthesis Study assessment, risk of bias assessment and data extraction were carried out independently by at least two reviewers. The primary outcome was bond failure rate. Results Thirteen studies involving 417 patients with 7184 teeth/ brackets were included. Two studies were considered to be at low risk of bias, 10 at high risk and one at unclear risk. Eleven studies compared the effects of self-etching primers (SEPs) with conventional etchants.

Five of these studies (three of split-mouth design and two of parallel design) reported data at the participant level, with the remaining studies reporting at the tooth level, thereby ignoring clustering/the paired nature of the data.

A meta-analysis of these five studies, with follow-up ranging from five to 37 months, provided low-quality evidence that was insufficient to determine whether or not there is a difference in bond failure rate between SEPs and conventional etchants (risk ratio 1.14; 95\% confidence interval $(\mathrm{Cl}) 0.75$ to $1.73 ; 221$ participants). The uncertainty in the $\mathrm{Cl}$ includes both no effect and appreciable benefit and harm. Subgroup analysis did not show a difference between split-mouth and parallel studies.

There were no data available to allow assessment of decalcification,

This paper is based on a Cochrane Review published in the Cochrane Library 2013, issue 11 (see www.thecochranelibrary.com for information). Cochrane Reviews are regularly updated as new evidence emerges and in response to feedback, and the Cochrane Library should be consulted for the most recent version of the review. participant satisfaction and cost-effectiveness. One study reported decalcification, but only at the tooth level.

Two studies compared two different SEPs. Both studies reported bond failure rate, with one of the studies also reporting decalcification. However, as both studies reported outcomes only at the tooth level, there were no data available to evaluate the superiority of any of the SEPs over the others investigated with regards to any of the outcomes of this review. No eligible studies were found evaluating different etching materials (eg phosphoric acid, polyacrylic acid, maleic acid), concentrations or etching times.

Conclusions We found low quality evidence that was insufficient to conclude whether or not there is a difference in bond failure rate between SEPs and conventional etching systems when bonding fixed orthodontic appliances over a five- to 37-month follow-up. Insufficient data were also available to allow any conclusions to be formed regarding the superiority of SEPs or conventional etching for the outcomes: decalcification, participant satisfaction and costeffectiveness, or regarding the superiority of different etching materials, concentrations or etching times, or of any one SEP over another.

Further well-designed RCTs on this topic are needed to provide more evidence in order to answer these clinical questions.

\section{Commentary}

The advent of predictable dental bonding has been central to the refinement and cosmetic improvement of fixed orthodontic appliances, ${ }^{1}$ with multi-banded systems now obsolete and bonding of molars increasingly preferred to banded attachments. In recent years the emphasis has increasingly shifted to streamlining bonding procedures, improving performance in moist environments and preventing demineralisation of enamel during treatment. ${ }^{2}$ Self-etch primers (SEPs) are now routinely used by up to $30 \%$ of practitioners in the US. ${ }^{3}$

Their potential advantages include a reduction in chairside time, lowered moisture sensitivity and reduced inventory requirements. Numerous clinical trials and one recent systematic review have addressed the relative merits of conventional acid-etching and SEPs4 in orthodontics.

This review augments previous evidence by attempting to compare outcomes with SEPs and the acid-etch technique and also by comparing various types of SEPs to one another. The review is clear and comprehensive and relates to an area of relevance to practising 
orthodontists. The main outcome measure was bond failure rate; secondary outcomes included demineralisation, patient satisfaction and costs.

It was notable that no assessment of chairside time was undertaken, given that reduced time requirement is an influential perceived advantage of SEPs.

Eleven studies involving comparison of SEPs and the acid-etch technique were identified. The majority, however, reported on tooth level rather than patient level data. Reporting failures for individual teeth fails to account for nesting of teeth within patients and quadrants leading to clustering effects. Erroneously handling clustered observations as independent may decrease standard errors and consequently produce artificially small p-values, increasing the likelihood of false positive results. Consequently, studies presenting tooth level analyses were not considered further, leaving five studies ranging in follow-up from five to 37 months in the meta-analysis.

In terms of bond failure rate, no significant difference between the techniques was found (risk ratio 1.14; 95\% confidence interval 0.75 to 1.73$)$.

The quality of the evidence was considered to be low with appreciable risk of bias in two of the primary studies and limited patient numbers. There were little data available concerning demineralisation, patient satisfaction and cost-effectiveness. Patient level data relating to comparisons of various types of SEP were not available.

Consequently, as is typical of many systematic reviews, the results are equivocal and expose a lack of relevant, high quality studies. The selection of enamel preparation technique, therefore, remains at the behest of the practitioner, as there does not appear to be sufficient evidence to suggest a difference in bond failure rates with acid-etching or SEPs.

The evidence base could be enhanced by conducting further research assessing bond failure rates on a patient level. It is also important that influential outcomes including demineralisation and cost-effectiveness are considered in future trials within this area.

\section{Practice points}

- There is limited evidence to suggest that no difference exists between orthodontic attachment failure rates with the acid-etch technique and self-etch primers.

- The choice of enamel preparation technique in orthodontics is dictated by individual preference in view of a lack of conclusive evidence.

Padhraig S Fleming

Barts and The London School of Medicine and Dentistry, Institute of Dentistry, Queen Mary University of London, UK

1. Newman GV, Snyder WH, Wilson CE Jr. Acrylic adhesives for bonding attachments to tooth surfaces. Angle Orthod 1968; 38: 12-18.

2. Eliades T. Orthodontic materials research and applications: part 1. Current status and projected future developments in bonding and adhesives. Am J Orthod Dentofacial Orthop 2006; 130: 445-451.

3. Keim RG, Gottlieb EL, Nelson AH, Vogels DS. 2008 JCO study of orthodontic diagnosis and treatment procedures. part 1: results and trends. J Clin Orthod 2008; 42: 625-641.

4. Fleming PS, Johal A, Pandis N. Self-etch primers and conventional acid-etch technique for orthodontic bonding: a systematic review and meta-analysis. Am J Orthod Dentofacial Orthop 2012; 142: 83-94.

Evidence-Based Dentistry (2014), 15, 48-49. doi: 10.1038/sj.ebd.6401025 